\title{
Coherent anti-Stokes Raman scattering performed on expanding thermal arc plasmas
}

\author{
R. F. G. Meulenbroeks, R. A. H. Engeln, J. A. M. van der Mullen, and D. C. Schram \\ Eindhoven University of Technology, Department of Physics, P. O. Box 513, 5600 MB Eindhoven, The Netherlands
}

(Received 1 November 1995)

\begin{abstract}
The expanding plasma emanating from a thermal arc plasma source that can be used for deposition of thin films is studied using laser spectroscopic techniques. The argon-hydrogen plasma is characterized by very fast recombination that cannot be explained by atomic processes. To explore this phenomenon, which has been related to wall association of hydrogen atoms and recirculation, CARS (coherent anti-Stokes Raman scattering) is performed on (argon-)hydrogen plasmas. The periphery of the plasma appears to be rich in hydrogen molecules, in accordance with the recirculation model. No highly rovibrationally excited states are detected in the periphery, in spite of the spectrometer's very good sensitivity $\left(0.1 \mathrm{~Pa} \mathrm{H}_{2}\right.$ at $\left.300 \mathrm{~K}\right)$. For the plasma, rotational and vibrational temperatures as well as absolute $\mathrm{H}_{2}$ densities are measured. A simple model for the observed (non-Boltzmann) rotational populations is developed.

PACS number(s): 52.25.-b
\end{abstract}

\section{INTRODUCTION}

Expanding thermal arc plasmas are used in a variety of applications, which include the deposition of diamond, diamondlike carbon, amorphous hydrogenated carbon, and amorphous hydrogenated silicon thin films [1]. When the thermal arc is operated either on pure hydrogen or on an argon-hydrogen mixture, it can be used as a particle source for hydrogen radicals or hydrogen positive and negative ions $[2,3]$. Fundamental research on expanding plasmas focuses on the study of argon-hydrogen mixtures. The principle parameters that have to be determined are particle densities (electrons, neutrals, and ions) and temperatures. To this end, active and passive spectroscopic techniques are employed. The results are interpreted by comparison with numerical models.

In this paper, we will summarize some of the properties of the argon-hydrogen expanding thermal arc plasma and describe in more detail the measurement of rovibrationally excited $\mathrm{H}_{2}$ molecules by CARS (coherent anti-Stokes Raman scattering) spectroscopy in and around the expanding plasma jet.

\section{EXPERIMENT AND DIAGNOSTICS}

The expanding thermal arc plasma has been described elsewhere $[4,5]$ and we will only give a brief description here. A subatmospheric (0.2-0.6 bar) plasma from a thermal arc (at high electron density) expands into a vacuum vessel, creating a plasma jet. The expansion direction defines the $z$ axis, with the origin at the expansion nozzle. Hydrogen can be added to the argon plasma in two ways: either by burning the arc on an argon-hydrogen mixture ("arc injection"), or by burning the arc on pure argon, and flushing hydrogen directly into the vessel ("vessel injection"). Basic plasma conditions are as follows: arc current $I_{\text {arc }}=45 \mathrm{~A}$, arc voltage $V_{\text {arc }}=50-100 \mathrm{~V}$, total flow $=3.5 \mathrm{SLM}$ (standard liters per minute), and background pressure $p_{\text {back }}=40-133 \mathrm{~Pa}$. The thermal arc is movable within a vacuum vessel without significantly changing the plasma. This greatly facilitates the axial and radial scanning of the plasma without moving the diagnostics.

Three diagnostics are applied to the plasma jet.

(i) Optical emission spectroscopy (OES). Passive spectroscopy in order to determine the excited level populations of hydrogen and argon. The setup is a fairly standard, calibrated, mirror-scanning system, described in detail in [5].

(ii) Thomson-Rayleigh scattering. A frequency doubled $\mathrm{Nd}$ :YAG (yttrium aluminum garnet) laser is used for this diagnostic. Photons are scattered off free and bound electrons in the plasma and detection of the scattered (Dopplershifted) radiation gives direct, local, and accurate information about electron and neutral densities and electron temperatures. The diagnostic has been described elsewhere [4]. A few important results will be discussed below.

(iii) Coherent anti-Stokes Raman scattering. In order to detect rovibrationally excited $\mathrm{H}_{2}$, a CARS diagnostic has been realized; it will be described below.

In order to get an impression of the processes that are important in the expanding jet, it is appropriate to discuss some of the basic properties (cf. [5-7].). The main characteristic of the argon-hydrogen jet is its anomalously fast recombination, as compared to the purely atomic argon case. As can be seen in Fig. 1, the electron density decreases by an order of magnitude, even for tiny admixtures of $\mathrm{H}_{2}$. This enormous ionization loss has been attributed to a combination of wall association and recirculation inside the vacuum vessel. In this process, hydrogen atoms associate to molecules at the vessel walls and are transported towards the plasma beam again by recirculation. For low hydrogen seed fractions, where the dominant ion is argon, the following reactions can occur:

$$
\mathrm{Ar}^{+}+\mathrm{H}_{2} \rightarrow \mathrm{ArH}^{+}+\mathrm{H},
$$

followed by

$$
\mathrm{ArH}^{+}+e \rightarrow \mathrm{Ar}+\mathrm{H}^{*},
$$

during which excited $\mathrm{H}$ atoms are formed. In the case of "arc injection," the $\mathrm{H}_{2}$ molecules necessary for these reactions do not all originate from the arc (where a high degree of disso- 


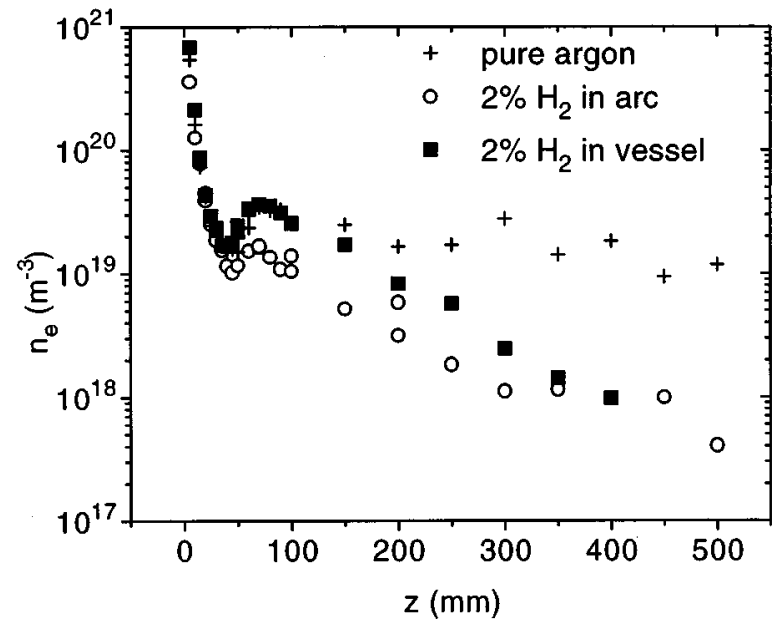

FIG. 1. Electron density vs axial position $(z)$ profiles of different expanding jets: pure argon, arc injection, and vessel injection.

ciation is assumed) but from the stainless steel vessel walls. A quasi-one-dimensional model [7] solving mass, momentum, and energy balances for the plasma particles confirms the existence of an external $\mathrm{H}_{2}$ input into the jet. The model predicts that this reentry flow penetrates the plasma around the shock position (i.e., around $z=40-70 \mathrm{~mm}$ in Fig. 1).

Absolute emission spectroscopy on the argon and hydrogen atomic systems shows that all the argon emission disappears for seed fractions above 7 vol.\%. As argon emission is due only to three particle recombination (low $T_{e}$, around 0.2 $\mathrm{eV}$ ), this means that the arc does not produce any argon ions for these seed fractions. The dominant ion therefore probably is $\mathrm{H}^{+}$for seed fractions above 7 vol.\% $[3,5,7]$.

Figure 1 actually represents the close investigation of the recirculation effect by a set of Thomson scattering experiments: a comparison is made between a pure argon jet, a $2 \%$ hydrogen-in-argon jet, and a pure argon jet with 2 vol.\% argon flushed directly into the vacuum vessel. The pure argon and "vessel injection" cases can be seen to clearly coincide up to $z=100$, i.e., the deviation starts after the shock region. The hydrogen-argon "arc injection" case shows a similar behavior, but has lower densities right from the start. The decrease after $z=100$ is remarkably similar for the "arc injection" and "vessel injection" cases. Eventually, a calculation shows that equal amounts of hydrogen are needed to explain the ionization loss after $z=100$. The ionization loss after the shock is thus thought to be totally determined by the background gas and the molecules present there. In order to scrutinize this phenomenon of very fast recombination through a molecular channel, a CARS diagnostic has been realized for the state-selective detection of hydrogen molecules.

\section{CARS SPECTROSCOPY}

\section{A. Theory}

The CARS process and signal generation have been described in a number of review articles (e.g. [8,9]) and we shall only give a brief account here. CARS is a coherent analog of Raman scattering with the advantage that the sig- nal is created as a coherent beam, thus greatly enhancing the sensitivity. This is the main reason why CARS was chosen as a diagnostic for our low pressure hydrogen-containing plasma. CARS has been chosen instead of other methods, such as resonance enhanced multiphoton ionization (REMPI) or VUV absorption, because its relatively easy in situ applicability in our case.

The CARS signal (at frequency $\omega_{0}=2 \omega_{1}-\omega_{2}$ ) is generated through the third-order nonlinear susceptibility $\chi^{(3)}$ of the probed molecule. The anti-Stokes power $\left(P_{3}\right)$ depends on the power of the pump $\left(P_{1}\right.$ at $\left.\omega_{1}\right)$ and Stokes $\left(P_{2}\right.$ at $\omega_{2}$ ) lasers as follows:

$$
P_{3}=K\left|\chi^{(3)}\right|^{2} P_{1}^{2} P_{2},
$$

where $K$ is a proportionality constant. The $\chi^{(3)}$ consists of a resonant and a nonresonant part: $\chi^{(3)}=\chi_{\mathrm{NR}}^{(3)}+\chi_{\text {res }}^{(3)}$. The resonant part is greatly enhanced when a Raman resonance is probed, i.e., when $\omega_{1}-\omega_{2}=\omega_{v, J}$, where $\omega_{v, J}$ is the frequency of a particular Raman transition. The resonant thirdorder susceptibility $\chi_{\text {res }}^{(3)}$ associated with a certain isolated $Q$ branch transition can be written as

$$
\chi_{\mathrm{res}}^{(3)}=K^{\prime} N\left[\rho_{v, J}-\rho_{v+1, J}\right](v+1) \frac{d \sigma}{d \Omega} h\left(\omega_{1}-\omega_{2}\right) .
$$

In this last equation, $K^{\prime}$ is a proportionality constant, $N$ is the total number of molecules, $\rho$ stands for the probability of occupation of a certain rovibrational state, $d \sigma / d \Omega$ is the (0-1) spontaneous Raman scattering cross section, and $(v+1)$ reflects the growth of this cross section with increasing vibrational quantum number. The form function $h\left(\omega_{1}-\omega_{2}\right)$ reflects the line shape. In our Doppler-limited regime, this function can be written as [10]

$$
\begin{aligned}
& h\left(\omega_{1}-\omega_{2}\right) \\
& \quad=\frac{1}{u \sqrt{(\pi)}} \int_{-\infty}^{+\infty} \frac{e^{-(\nu / u)^{2}}}{\omega_{v, J}-\omega_{1}+\omega_{2}-i \gamma_{v, J}+\left(\omega_{1}-\omega_{2}\right) \nu / c} d \nu,
\end{aligned}
$$

where $\omega_{v, J}$ is the frequency of the transition considered and $\gamma_{v, J}$ its full width at half maximum (FWHM), $u$ is the root mean square velocity, $u=\sqrt{2 k T / m}, \nu$ is the molecule's velocity, and $c$ is the speed of light. In practice, $\gamma_{v, J}=0$ can be taken in our case, where pressures are well below $500 \mathrm{~Pa}$. In this case, the linewidth and shape is determined by the Doppler effect $\left(0.09-0.15 \mathrm{~cm}^{-1} \mathrm{FWHM}\right)$, at temperatures ranging from $500-2000 \mathrm{~K}$. The dye linewidth is around 0.07 $\mathrm{cm}^{-1}$, the pump laser can be considered monochromatic $\left(0.005 \mathrm{~cm}^{-1}\right)$. In our case of well-separated lines (because of the large molecular constants of $\mathrm{H}_{2}$ ), we do not have to worry about interfering lines: as the surface of a line can be very well determined, the spectral line shape does not have to be considered. Doppler broadening, however, does have an effect, as it decreases the peak height of a line, which impedes the measurement of very faint lines.

With an appropriate calibration on hydrogen gas, absolute values of $N\left[\rho_{v, J}-\rho_{v+1, J}\right]$ can be obtained, using the above description. If a number of transitions is monitored, absolute values of the population densities of individual rovibrational 


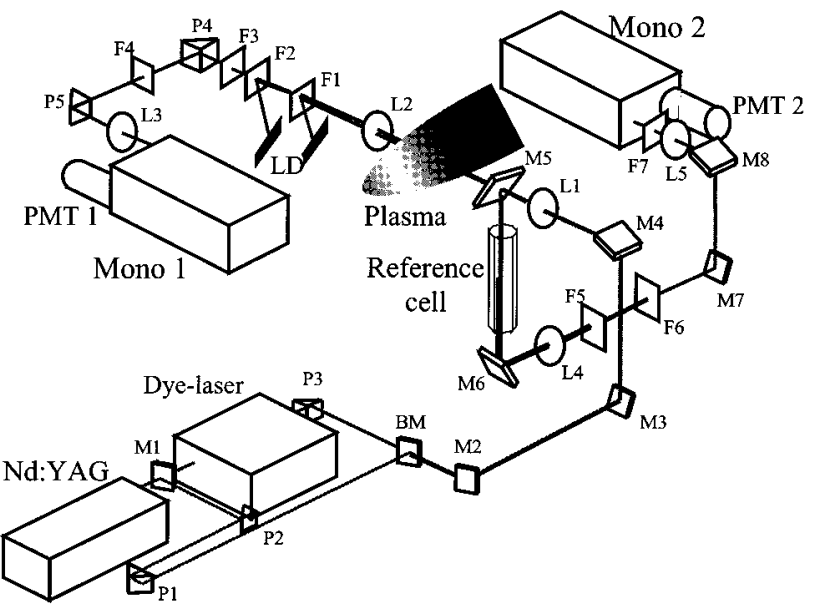

FIG. 2. The CARS spectrometer at the Eindhoven University of Technology. A Nd:YAG laser is used to generate the pump beams and to pump the dye laser $\left(M_{1}\right.$ : a 70/30 beam splitter). The beams are in a BOXCARS arrangement (inset): a dichroic beam combiner $(\mathrm{BM})$ is used to make the red beam coincide with one of the green beams. A system of mirrors $\left(M_{2}-M_{8}\right)$ takes the beams up to the experiment (the vacuum vessel is at a height of approx. $3 \mathrm{~m}$ ). $M_{5}$ is a flat quartz plate used to split off $10 \%$ of the laser energy, which is led through the reference cell. $P_{1}-P_{5}$ are prisms $\left[P_{1}\right.$ is used to create a time delay line to compensate for the time loss (several ns) in the dye laser], $L_{1}-L_{5}$ are lenses. The CARS lenses $\left(L_{1}, L_{2}\right.$, and $L_{4}$ ) have a focal length of $1 \mathrm{~m}$. The CARS signal is filtered from the green beam by a set of filters $\left(F_{1}-F_{3}\right.$ and $\left.F_{5}-F_{6}\right) . F_{4}$ represents an eventual neutral density filter. LD designates laser dumps, PMT designates a photomultiplier tube. All high power optics are in suprasil quality quartz.

states can thus be calculated. We shall return to this later when we describe the measurements procedure.

\section{B. The CARS spectrometer}

The experiment is depicted in Figs. 2 and 3. An injection seeded Nd:YAG laser (Quanta Ray GCR230, $300 \mathrm{~mJ} /$ pulse at $532 \mathrm{~nm}, 50 \mathrm{~Hz}$, single longitudinal mode) is used for the $\omega_{1}$ beam (bandwidth $0.005 \mathrm{~cm}^{-1}$ ); it also pumps a Quanta Ray PDL3 dye laser (bandwidth around $0.07 \mathrm{~cm}^{-1}$ ). The

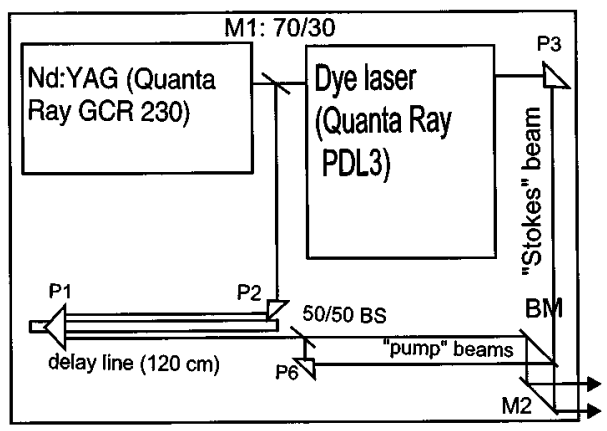

FIG. 3. The CARS laser table. The commercial lasers are positioned on an optical table, supported by concrete blocks. This figure shows in some detail the delay line (to correct for the delay in the dye laser), the BOXCARS beam splitter $\left(P_{6}\right)$, and the beam combiner $(\mathrm{BM})$, which aligns the red and green beams. dye liquid is a mixture of DCM and LDS698 (exciton) solved in methyl alcohol in a mass ratio of $3: 1$; it delivers conversion in the region of $660-700 \mathrm{~nm}$. Eventually, the used laser output powers are around 10-20 mJ/pulse for the green and around $0.5 \mathrm{~mJ}$ for the red. The $\omega_{1}$ and $\omega_{2}$ beams are in a crossed beam arrangement (BOXCARS), and the detection volume has dimensions of about $20 \times 0.18 \mathrm{~mm}$. A small part $(10 \%)$ of the beams is split off after the CARS lens $(f=1 \mathrm{~m})$ by a $(\lambda / 20)$ flat quartz plate and led through a reference cell (1m length) containing 7 bars of argon. The nonresonant CARS signal created here is used as a reference to cancel out shot-to-shot variations in beam overlap and laser power, as well as variations in the dye laser output at different wavelengths. Extreme care has been taken to make the reference and main branches as identical as possible, especially up to the position of CARS signal generation. The correct pressure dependence (intensity proportional to the square of the pressure) has been verified in the reference cell and vacuum vessel.

After collimation by a second lens, the blue anti-Stokes signals are separated from the green pump beam by filtering. Both main and reference CARS signals are guided through identical $1 \mathrm{~m}$ Czerny-Turner monochromators (Hilger-Engis Monospek 1000) for separation of signal from residual green light. This filtering is very effective: no signal is obtained if either the green or the red beam is dumped before the CARS lens $\left(L_{1}\right)$. Signals are detected using fast photomultiplier tubes (Hamamatsu R1355, cooled to $-25^{\circ} \mathrm{C}$ ), using a tapered bleeder resistor network in order to insure good pulse linearity. When the CARS signal is very strong (e.g., in the case of a $v=0, J=1$ line), a set of calibrated neutral density (ND) filters is used to attenuate the signal. A fast, multichannel, gated ADC (LeCroy 2249A) simultaneously integrates the main and reference PM tube outputs during a 150 ns time interval, gated by the $Q$-switch synchronization signal generated by the Nd:YAG laser unit.

The stepping of the dye laser and the monochromators, as well as the processing and storage of the ADC outputs, is controlled by a 486 PC. A measurement is performed by scanning the dye laser in steps of $0.015 \mathrm{~cm}^{-1}$ over the different $\mathrm{H}_{2} Q$ branch resonances, taking 50 or 100 measurements at each point. Both monochromators are set to the correct position for detection of the generated anti-Stokes signal. The main signal is immediately (pulse-by-pulse) divided by the reference signal and stored for further processing. The program automatically skips the intervals between Raman resonances, since these are relatively far apart in hydrogen.

For a better grasp of what is passing inside the vacuum vessel (which is not easily accessible in our case), a CCD camera system is installed on which an attenuated second reflection (after lens $L_{1}$ ) of pump and dye lasers is focused. With appropriate filtering, a black-and-white picture can be obtained that is representative of the situation in the main CARS generation volume, showing the beam overlap. This greatly facilitates the alignment of the setup and makes possible a check of the alignment while measuring.

\section{Tests and measurement procedures}

Before turning to measurements, the spectrometer has been carefully tested and calibrated, focusing on aspects such 


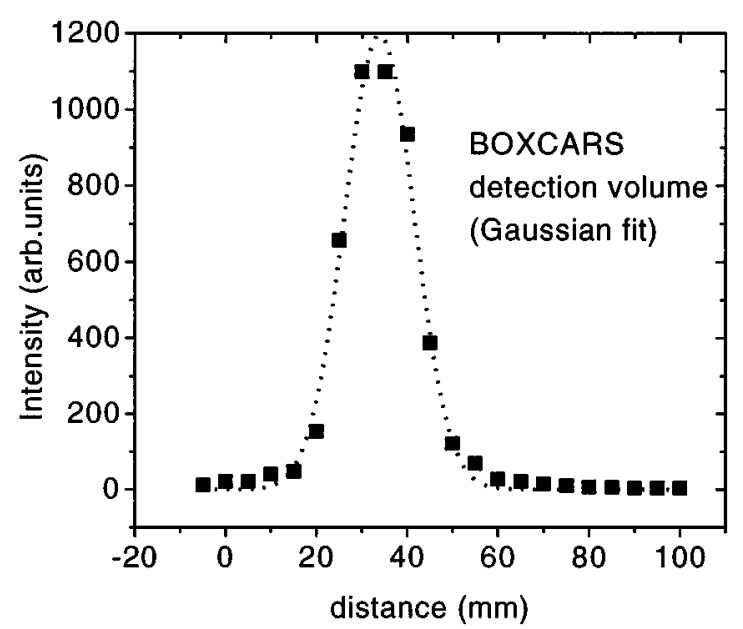

FIG. 4. A measurement of spatial resolution in the reference branch by probing the nonresonant CARS signal generated in a 2 $\mathrm{mm}$ thick quartz plate at different positions.

as linearity (of the detection system, especially the PM tubes and the ADC), spatial resolution, saturation effects, and detection limits. The linearity of the detection system is checked using calibrated ND filters and proves to be excellent (with a few \%).

Spatial resolution is measured in the reference branch. After removal of the argon cell a $2 \mathrm{~mm}$ quartz plate on a mounting is placed in the focal point of the laser beams. The signal now detected in the reference branch is due mainly to nonresonant CARS generated in the quartz. By moving the thin quartz plate through the detection volume, a measurement of the detection volume is basically performed. The result is shown in Fig. 4. With a beam separation of $35 \mathrm{~mm}$ at the CARS lens (leading to an angle between the BOXCARS beams of $1^{\circ}$ ), a spatial resolution of approximately $20-30 \mathrm{~mm}$ is achieved in the longitudinal direction. In the plane perpendicular to the laser beams, the resolution is determined by the waist of the focus, around $180 \mu \mathrm{m}$.

The phenomenon of saturation in CARS is particularly important as it limits the laser powers that can be applied without significantly changing the population distribution in the plasma. This phenomenon has been studied extensively by Péalat et al. [11]. The process that causes redistribution among the probed states is stimulated Raman scattering (SRS [8]). To estimate the importance of this effect, one has to measure the power dependence of the signal strength, in order to check the validity of Eq. (3). This has been done using beam splitters in the first part of the green beam (i.e., just after the 70/30 beam splitter in Fig. 3). Measuring the power dependence is very cumbersome, as the removal of a beam splitter makes a realignment necessary. Nevertheless, the results are reproducible and Fig. 5 shows the surface of the measured $v=0, J=1$ peak (designating the transition by the lower level) vs $P_{1}^{2} P_{2}$. Although we are able to measure only a few points, the saturation behavior is very clear for the higher laser powers.

Using a computer code by Péalat et al. at ONERA, a simulation has been performed for the saturation behavior. The procedure is completely analogous to the one described for $\mathrm{N}_{2}$ in [11] and will not be fully discussed here. Using

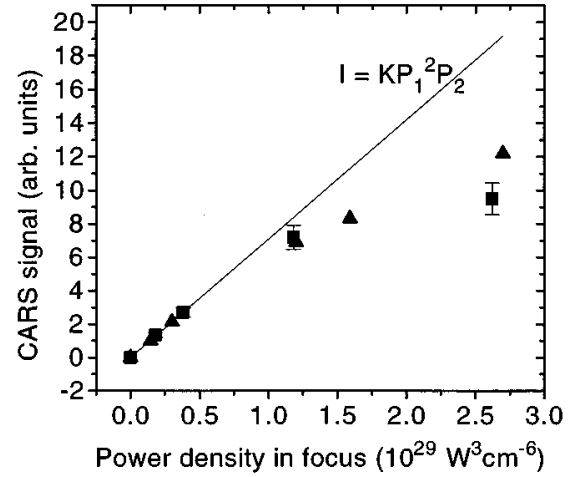

FIG. 5. Power dependence measurement of the $v=0, J=1 Q$ line. The triangles represent a model fit [12], where scaling has been performed by adjusting the focal waist diameter. The ideal behavior $\left(I=K P_{1}^{2} P_{2}\right)$ is observed for low powers.

molecular data for $\mathrm{H}_{2}$ and dye and pump powers in the vessel, a fit could be made to the points in Fig. 5 (i.e., for $v=0, J=1)$, assuming a nine-mode dye laser $(\approx 0.07$ $\left.\mathrm{cm}^{-1}\right)$. Of course, the actual power density in the vessel is not known, because the actual beam waist can only be accurately calculated for the case of a perfect Gaussian laser beam. In the present case this is not possible, as the PDL3 dye laser delivers no Gaussian profile, whereas the injection seeded Nd:YAG laser has a reasonably Gaussian profile ( $>90 \%$ far field). The procedure therefore relies on adjusting the laser power densities, varying the value of the beam waist, to fit the model calculations to the experimental values. With a (reasonable) beam waist of around $180 \mu \mathrm{m}$, a good fit has been obtained, shown in Fig. 5. In principle, this makes possible the correction of saturated data (as the dependence of the saturation on the $v$ quantum number is known [11]), but in practice we prefer to stay in the unsaturated region with pump and dye powers. However, some uncertainty is always present as it is very difficult to check on saturation behavior of higher excited levels. Nevertheless, we shall neglect the effects of saturation in the following.

In order to test the response of the entire system, so-called "argon-argon" measurements are performed before and after each measurement session. Here, the vessel is filled with 60 mbars of argon and a measurement of the nonresonant CARS signal on argon performed scanning the entire $\mathrm{H}_{2}$ $(v=0-4)$ range, i.e., Raman shifts in the range 4162-3000 $\mathrm{cm}^{-1}$. Since reference and main branches should show the same behavior now, their ratio should remain constant-all uncertainties concerning beam overlap, dye laser output, etc. should be symmetrized. This has been checked to be the case within $10 \%$ for every measurement.

A calibration has to be performed in order to obtain absolute values for the densities. Room temperature $\mathrm{H}_{2}$ at a pressure of $70 \mathrm{~Pa}$ in the vessel is used for calibration: four levels are used, even though one would in principle suffice. Calibrations are also performed before and after each measurement session.

\section{CARS RESULTS}

\section{A. Measurement and processing}

A typical measurement is shown in Fig. 6. It shows the 


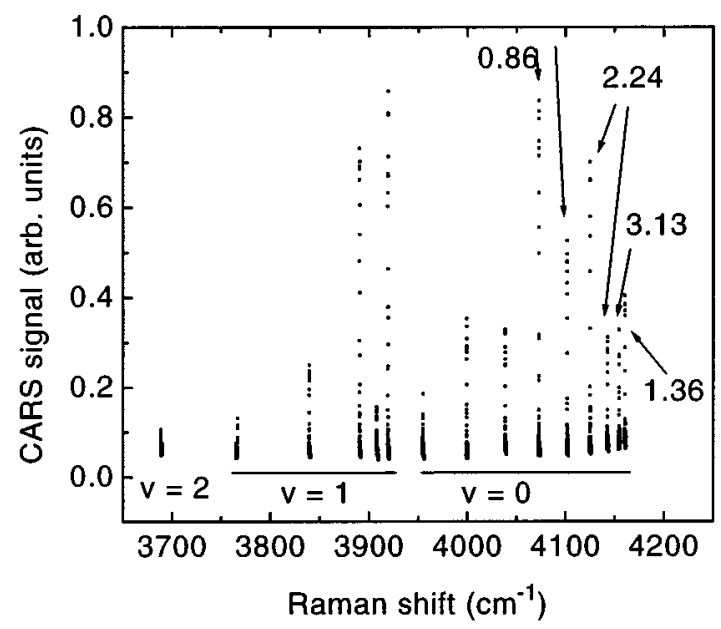

FIG. 6. A typical measured CARS spectrum in a full $\mathrm{H}_{2}$ plasma. $(z=150 \mathrm{~mm})$. The values $n d$ of the attenuating ND filters are indicated (the attenuation equals $10^{\text {nd }}$ ).

distinct Raman resonances for $v=0, v=1$, and $v=2$ levels. In full $\mathrm{H}_{2}$, measurements could be taken up to $v=2, J=5$. The values of the attenuating ND filters are indicated. Processing takes place by determining the surface under each line and correlating these surfaces to those in the $\mathrm{H}_{2}$ calibration, using Eqs. (3) and (4). Corrections are made for the $(v, J)$ dependence of the Raman cross section using data from Long [13] and others [14,15]. In $\mathrm{H}_{2}$, we can use the isolated line approximation. In this manner, absolute density differences are obtained that have to be converted to densities using certain assumptions. In our case, we assumed the density of the level above the one that can just be measured to be negligible, so we assume the following for $v=2$ levels: $n(v=2, J)-n(v=3, J) \approx n(v=2, J)$. The error is probably not larger than a few percent under our conditions. For lower levels, we follow Eq. (4):

$$
n(v=i, J)=n_{\text {measured }}(v=i, J)+n(v=i+1, J) .
$$

In the end, absolute densities can be obtained with a total accuracy of $10 \%$. The detection limit of the system is around $0.1 \mathrm{~Pa} \mathrm{H}_{2}(300 \mathrm{~K})$; during the measurements, densities per statistical weight as low as $2 \times 10^{17} \mathrm{~m}^{-3}$ have been measured for $v=2$. Boltzmann plots of these measurements will be shown later on.

\section{B. Results for argon-hydrogen mixtures}

The first aim of the CARS measurements is to scrutinize the phenomenon of very fast recombination. Therefore CARS has been performed on 10 and 50 vol. $\% \mathrm{H}_{2}$ in $\mathrm{Ar}$ mixtures (vessel and arc injection, $I_{\text {arc }}=55$ A, total flow 3.5 SLM, background pressure $133 \mathrm{~Pa}$ ). In all cases the view is confirmed that the background gas contains lots of relatively cold $\left(T_{\text {rot }}=400 \mathrm{~K}, T_{\text {vib }}=1100 \mathrm{~K}\right)$ hydrogen molecules. In fact, the relative partial hydrogen pressure in the periphery of the plasma appears to be equal to the seed fraction: an $x$ vol.\% seed fraction results in a partial hydrogen pressure that is equal to $x \%$ of the total background pressure. This holds for both vessel and arc injection. No highly excited vibra-
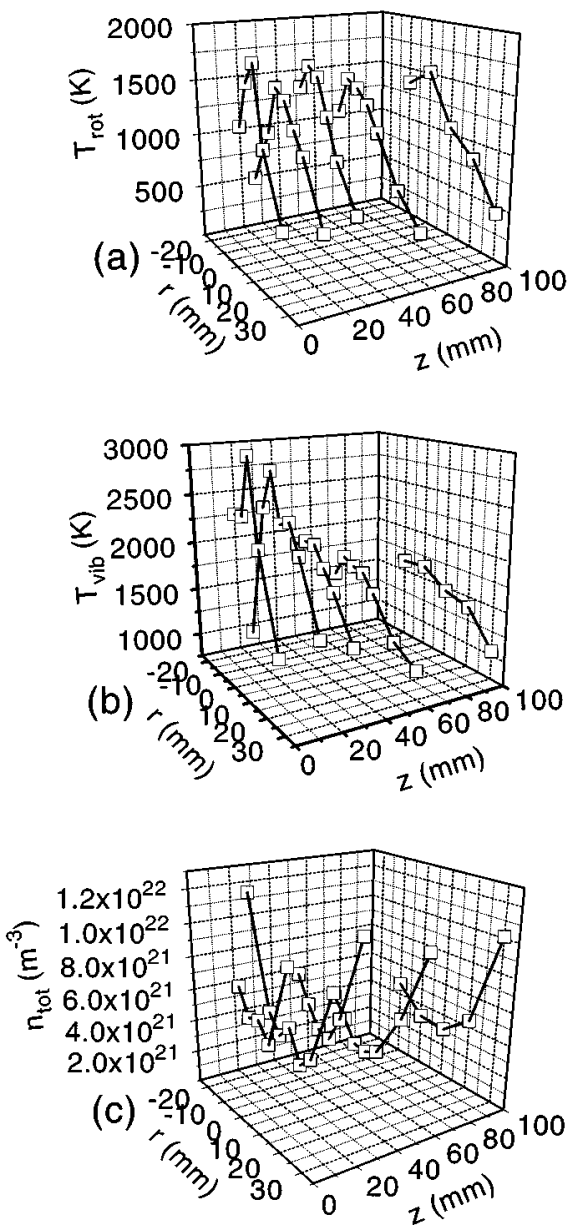

FIG. 7. CARS measurements on a 50 vol. $\% \mathrm{H}_{2}$ in Ar plasma (arc injection). (a) Rotational temperatures in the $v=1$ state; (b) vibrational temperatures calculated using the $J=5$ states; (c) total density profiles. The radial profiles are taken at axial positions $z=10,20,40,60$, and $100 \mathrm{~mm}$.

tional states were detected in the periphery of the plasma, within the detection limits. Other $\mathrm{H}_{2}$ measurements in Ar are to be presented in detail in a separate letter [16].

Figure 7 gives the results for the $50 \mathrm{vol} . \% \mathrm{H}_{2}$ in argon plasma. In these plots, the rotational temperatures are taken to be the temperatures in the $v=1$ state, as the $v=0$ population suffers from some contributions by the cold background gas. This is the case only for a $133 \mathrm{~Pa}$ background pressure, as the beam diameter in this case is of the same order as the spatial resolution along the laser beams (i.e., a few $\mathrm{cm}$ ). Therefore, the radial dependencies should only be viewed upon as an indication. For the same reason, the vibrational temperatures are calculated using the $J=5$ levels of $v=0$ and $v=1$ (we can only measure these vibrational states in this plasma), as comparing the $J=1$ levels (for example) would lead to an underestimation of $T_{\text {vib }}$. This is caused by the relatively large amount of $(v=0, J=1)$ states in the cold background which are in the detection volume. The densities are taken to be the sum of all measured states, making appropriate corrections for states that cannot be measured. The poor spatial resolution means the construction of a pressure plot is not very useful: the measured density includes both hot and cold parts, but if the temperature of the hot part (i.e., 
the $v=1$ data) is assigned to the total density, the calculated pressures in the plasma jet would be too high. However, the partial pressure in the periphery (where there are no special demands on spatial resolution) appears to be equal to $50 \%$ of the background pressure (i.e., $69 \mathrm{~Pa} \pm 10 \%$ ), which is equal to the seed fraction of $\mathrm{H}_{2}$ in Ar. Note that this is not a trivial result, as the arc plasma is characterized by a high dissociation degree.

In the following, the background pressures are lower $(\leqslant 40 \mathrm{~Pa})$, and in these cases the spatial resolution along the laser beams appears to be adequate, at least for distances from the nozzle larger than $20 \mathrm{~mm}$ or so.

\section{Results for pure hydrogen plasmas}

CARS measurements have also been performed on plasmas in pure hydrogen, both with and without a magnetic field to confine the plasma. The plasma conditions are as follows: no magnetic field: $I_{\text {arc }}=37.5$ A, flow: 3.5 SLM, $p_{\text {back }}=40 \mathrm{~Pa}$; with magnetic field: $I_{\text {arc }}=37.5$ A, flow: 2 SLM, $p_{\text {back }}=20 \mathrm{~Pa}, B$ field: $1 \mathrm{kG}$. The $B$ field can be considered homogeneous over the entire length of the plasma. In the latter case, the electrons are magnetized, whereas the ions are not. The magnetized plasma has been studied in view of the possible high density of negative ions there [17]. As negative ions may be formed by the well-known dissociative attachment reaction

$$
\mathrm{H}_{2}^{(v, J)}+e^{-} \rightarrow \mathrm{H}^{+}+\mathrm{H}^{-},
$$

where highly excited $\mathrm{H}_{2}$ is necessary [19] to make the reaction efficient, studying the $\mathrm{H}_{2}^{(v, J)}$ population is essential.

The results are shown in Fig. 8 for the nonmagnetized plasma, and in Fig. 9 for the magnetized case. Shown are typical Boltzmann plots for the $v=0$ to $v=2$ states, and axial dependencies for the major parameters, $T_{\text {rot }}, T_{\text {vib }}, p$, and $n_{\text {tot }} . T_{\text {rot }}$ has been taken from the $V=1$ data. The rotational temperatures are equal for $v=0, v=1$, and $v=2$, as can be seen from the Boltzmann plot in Fig. 8(b), at $z=150 \mathrm{~mm}$. $T_{\text {vib }}$ has been calculated using the $J=3$ or (whenever possible) the $J=5$ states.

It may be useful to ponder a little on the spatial resolution and the reasons we have to believe that it is sufficient in these lower pressure cases (i.e., the reason why we can believe the $v=0$ rotational distributions to be virtually undisturbed by the periphery gas). In the argon-hydrogen measurements under $(B)$, the $v=0, J=0-3$ levels always show a rotational temperature that is unrealistically low, around $400 \mathrm{~K}$, in clear disagreement with $T_{\text {rot }}$ in $v=1$ (Fig. 7). The $T_{\text {rot }}$ of $400 \mathrm{~K}$ corresponds to the temperature measured in the peripheral gas. So in this case, the spatial resolution along the laser beams is inadequate: some cold background gas is measured along with the hot plasma. In the lower pressure case, however, the $v=0, J=0-5 T_{\text {rot }}$ 's are in good agreement with the $v=1$ and $v=2$ data, at least for downstream distances larger than $20 \mathrm{~mm}$. Therefore, we assume the rotational distributions in Figs. 8 and 9 to be "real," and that they contain no artifacts.

From Figs. 8 and 9, it is worthwhile to note that, even per statistical weight, the population in a high rotational level of $v=0$ can be as large as the population in a lower rotational level in $v=1$, at the same energy. With the advantage of a larger statistical weight, more energy can be carried by rotations than by vibrations in our plasma. This is an important observation. It distinguishes these expanding plasmas from many other plasma sources, where the rotational temperature is usually very low compared to the vibrational temperature (e.g., [10]). In the latter cases, research therefore often focuses on the vibrational population distribution. The measurements in this section can serve as an example for a situation where the rotational population is very important. In this respect, our situation is somewhat similar to combustion research [18].

It follows from the pressure plots in Figs. 8 and 9 that the dissociation degree in the plasma jet must be minimum, as the measured $\mathrm{H}_{2}$ pressures account for the total background pressure. Note that high pressures are measured near the nozzle. This could be an artifact: we can expect to measure some background gas there (the beam diameter is still small) and still ascribe a high $(v=1)$ rotational temperature to the total measured $\mathrm{H}_{2}$ density at these positions. This would lead to an overestimation of the pressure.

The fact that the measured dissociation degree in the plasma is so low is very surprising, as power input measurements show that the arc must have a dissociation degree of around $70 \%$ for an arc current of $37.5 \mathrm{~A}$ [20]. This has been more or less confirmed by depolarization Rayleigh scattering measurements very close to the arc [2]. A further indication follows from the analysis of the rotational populations, measured by CARS, in the following section. It is our opinion that only an effective wall-association process in combination with a recirculation flow can explain these results $[5,7,16]$.

Furthermore, no great dissimilarities are obvious from a comparison between Fig. 8 and Fig. 9. Indeed, the hydrogen molecular population hardly seems to be influenced by the fact that a totally different plasma is present in the magnetized case. Especially the absence of highly excited vibrational states in the magnetized case makes less probable the occurrence of mechanism (7) as a main $\mathrm{H}^{-}$production channel. If $\mathrm{H}^{-}$is present in large amounts, a different mechanism might be necessary, namely, the formation through Rydberg or highly (electronically) excited $\mathrm{H}_{2}$, as proposed by Garscadden et al. in [21].

\section{ROTATIONAL RELAXATION MODEL}

An attempt has been made to explain the non-Boltzmann rotational distributions in Fig. 8 by considering hot $\mathrm{H}_{2}$ gas (i.e., the $30 \%$ that is not dissociated) flowing out of the arc, relaxing to an infinitely large, cold $\mathrm{H}_{2}$ background. During the relaxation by rotational-translational inelastic collisions ( $R-T$ collisions), higher states relax more slowly. The rate for a given $\Delta J=L$ can be expressed in the rate for relaxation for $L \rightarrow 0$, as has been established by DePristo et al. [22]:

$$
\begin{aligned}
k\left(J_{i} \rightarrow J_{f}\right)= & g_{J_{f}} \exp \left(\frac{E_{J_{i}}-E_{J_{>}}}{k T_{\text {gas }}}\right) \\
& \times \sum_{L}\left(\begin{array}{ccc}
J_{i} & L & J_{f} \\
0 & 0 & 0
\end{array}\right)^{2} g_{L}\left|A_{L}^{J_{>}}\right|^{2} k(L \rightarrow 0),
\end{aligned}
$$

where $J_{i}$ and $J_{f}$ are the initial and final $J$ values, $J_{>}$is the largest of the two. The exponential follows from micror- 

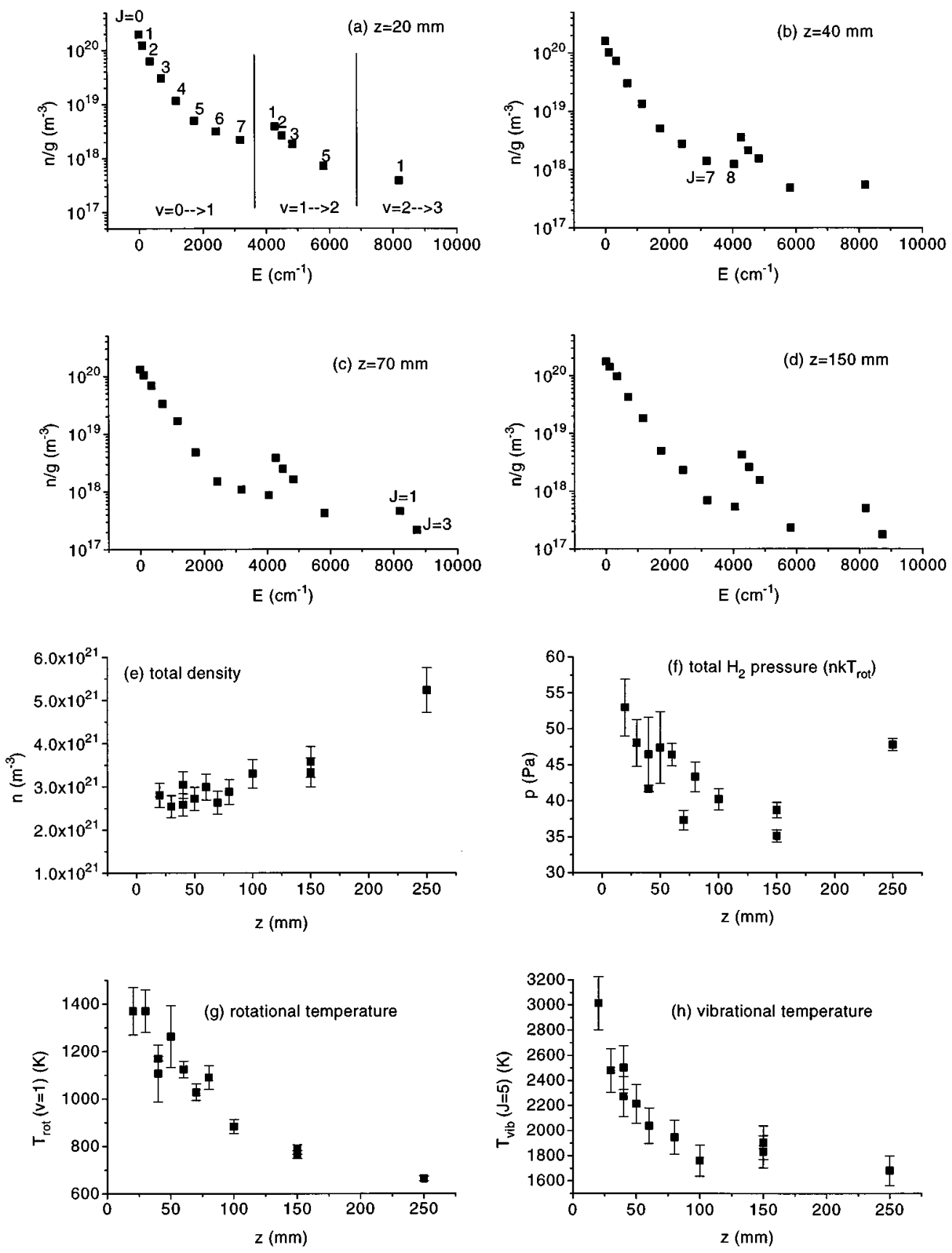

FIG. 8. CARS results for a full hydrogen plasma: Boltzmann plots for different axial positions (a)-(d), and axial dependencies of the axial position is denoted by $z, E$ is the energy, and $n / g$ represents the density per statistical weight.

eversibility, the $3 j$ symbol expresses the recoupling of $J_{i}$ with $L$ to form $J_{f}, g$ denotes the statistical weight, and $A_{L}^{J_{>}}$denotes an "adiabaticity" factor, defined by

$$
A_{L}^{J_{>}}=\frac{1+\tau_{L}^{2} / 6}{1+\tau_{J_{>}}^{2} / 6},
$$

where $\tau_{J}=2 \pi T_{c} / T_{J}$, with $T_{c}$ the collision time and $T_{J}$ the characteristic time of rotation for a given $J$. The adiabaticity factor accounts for the effectivity of momentum exchange for given collision and rotation times. It is close to 1 for our conditions.
The above gives the framework for a very simple model to explain the rotational distributions. For a flux of 3.5 SLM (i.e., $1.4 \times 10^{21} \mathrm{~s}^{-1}$ ), a $70 \%$ dissociation degree, an estimated velocity of $5 \times 10^{3} \mathrm{~m} / \mathrm{s}$ (see below), and a beam diameter at $z=20 \mathrm{~mm}$ of $15 \mathrm{~mm}$, we obtain a concentration of approximately $5 \times 10^{20} \mathrm{~m}^{-3}$ at $z=20 \mathrm{~mm}$. The total $\mathrm{H}_{2}$ density at that position (measured by CARS, Fig. 8) is around $2.8 \times 10^{21} \mathrm{~m}^{-3}$. In the model, we use a hot density of $5 \times 10^{20} \mathrm{~m}^{-3}$, and a cold $\mathrm{H}_{2}$ density of $2.3 \times 10^{21} \mathrm{~m}^{-3}$. A set of simple differential equations is constructed for the first 14 levels in the $v=0$ manifold. The system looks as follows: 

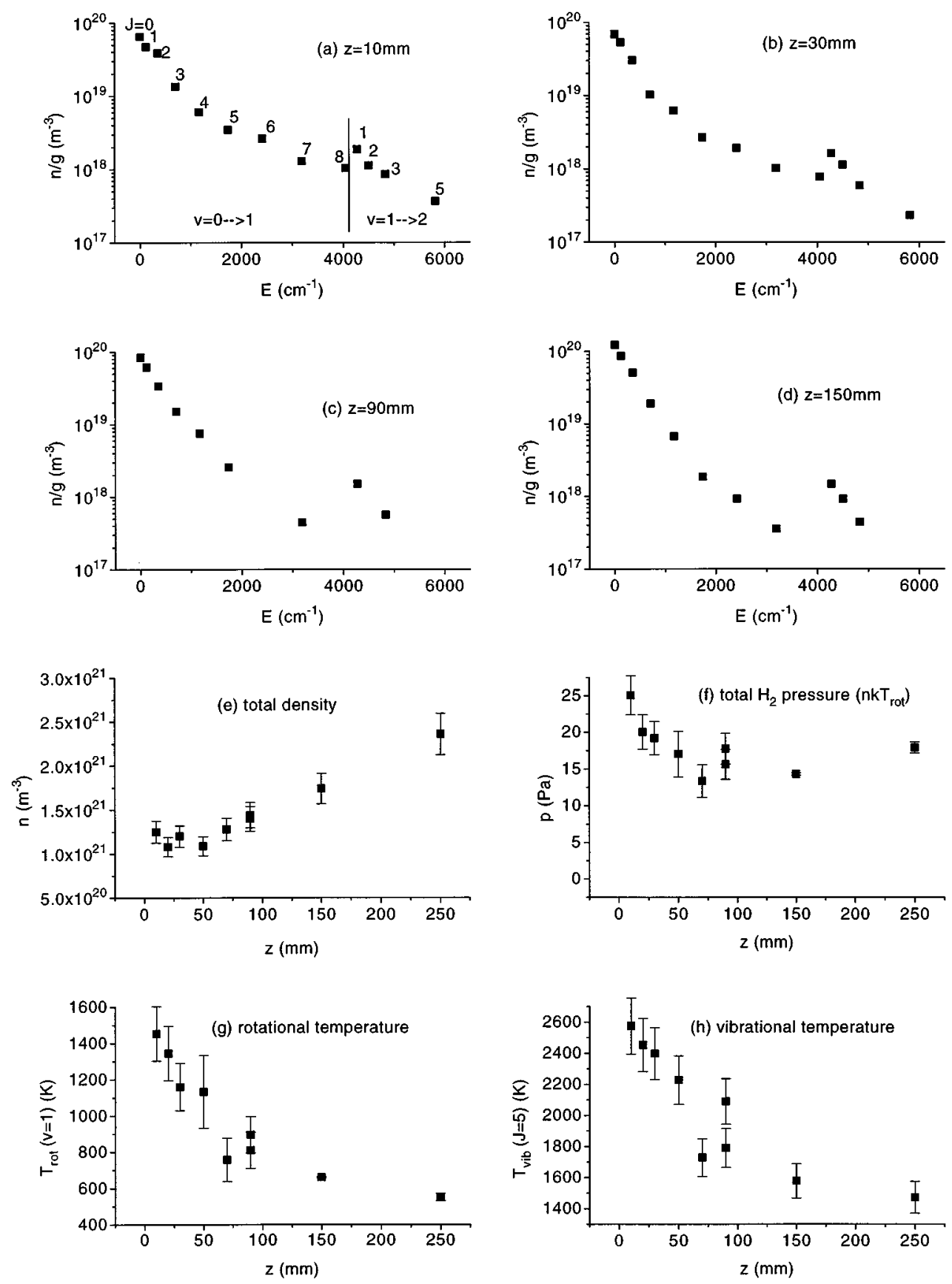

FIG. 9. CARS results for the magnetized hydrogen plasma beam. Boltzmann plots for different axial positions (a)-(d), and axial dependencies for total density $n$ (e), total partial $\mathrm{H}_{2}$ pressure $p$ (f), $T_{\text {rot }}\left(\mathrm{g}\right.$ ), and $T_{\text {vib }}(\mathrm{h})$.

$$
\begin{aligned}
u \frac{d n_{0}}{d z}=N\left(n_{2} k_{2,0}-n_{0} k_{0,2}\right), & u \frac{d n_{13}}{d z}=N\left(n_{11} k_{11,13}-n_{13} k_{13,11}\right), \\
u \frac{d n_{1}}{d z}=N\left(n_{3} k_{3,0}-n_{1} k_{1,3}\right), & u \frac{d n_{14}}{d z}=N\left(n_{12} k_{12,14}-n_{14} k_{14,12}\right),
\end{aligned}
$$

$\left.u \frac{d n_{i}}{d z}=N\left(n_{i+2} k_{i+2, i}+n_{i-2} k_{i-2, i}\right)-n_{i}\left[k_{i, i+2}+k_{i, i-2}\right]\right)$

$(2 \leqslant i \leqslant 12)$ where $k_{i, j}$ denotes a rate [calculated using (8)], $N$ is the density of collision partners (i.e., the cold $\mathrm{H}_{2}$ ), and $n_{i}$ are the densities for each rotational level. We only consider $\Delta J=2$ collisions, because of nuclear spin conservation for $\mathrm{H}_{2}$ (ortho and para $\mathrm{H}_{2}$ conservation). Larger (even) $\Delta J$ steps are 
neglected [23]. The speed of the jet is represented by $u$, which has been cast in the following (assumed) form:

$$
u(z)=u_{0} \exp (-z / l),
$$

where $u_{0}$ is the speed at the origin (the nozzle), taken to be $7 \times 10^{3} \mathrm{~m} / \mathrm{s}$ (calculated using pressure and flow data from a similar experiment) and $l$ is a characteristic length, taken to be $70 \mathrm{~mm}$. The expanding jet collides with the background gas (at $600 \mathrm{~K}$ ) with velocity $u(z)$, and both the thermal energy and the translational energy are taken into consideration in the calculation of the rate constant $k(2 \rightarrow 0)$ at a certain energy. The cross sections for $2 \rightarrow 0$ at temperatures in the range $500-4000 \mathrm{~K}$ are calculated using data from Green [24]. A second-order polynomial fit delivers for this temperature range:

$$
k_{2 \rightarrow 0}(T)=-1.55 \times 10^{-18}+2.65 \times 10^{-21} T+5.5 \times 10^{-25} T^{2},
$$

where $T$ is the collision energy in $\mathrm{K}$.

The system is solved using MAPLE [25] with a Boltzmann distribution at $3000 \mathrm{~K}$ (which seems to be a reasonable value for hot molecules leaving the arc) as a $z=0$ boundary condition.

Figure 10 shows the results for the model, together with the measurements in a pure hydrogen plasma (no magnetic field) at two different positions. In using one model population to describe the populations at two positions, we implicitly assume that the increase in area of the primary beam (hot population) is balanced by a decrease in velocity, because the hot and cold model populations remain the same. The total density at the two $z$ positions is about equal (Fig. 8). In spite of the many assumptions, the model appears to describe the measurements very well. Because CARS "sees" both the hot and cold populations, the temperature in the lower part of the rotational manifold seems rather low, whereas the tail is hot. As the tail slowly relaxes, the total distribution becomes more and more Boltzmann-like.

The dissociation degree of $70 \%$ inside the arc is a necessary condition to obtain a good agreement. To illustrate this, a few runs with the program have been performed using a very low dissociation degree (10\%) in the arc. In this case, the majority of the arc output flux consists of molecules with a temperature of $3000 \mathrm{~K}$, relaxing to a background gas at 600 $\mathrm{K}$ (just as in the above). Using the same speed profile and the same expressions for the rate constants, results are obtained that deviate significantly form the experimental findings (Fig. 10). Apparently, the large amount of hot molecules does not relax quickly enough in this case. This is a further indication that the arc does dissociate to a large extent, even though the model is probably not accurate enough to distinguish between a 70 or $80 \%$ arc dissociation degree.

\section{CONCLUSIONS}

The expanding argon-hydrogen plasma jet is characterized by an anomalously fast recombination that can be explained by molecular channels involving hydrogen molecules. This is clearly indicated by the Thomson scattering measurements. CARS measurements show that large amounts of hydrogen molecules are indeed present in the
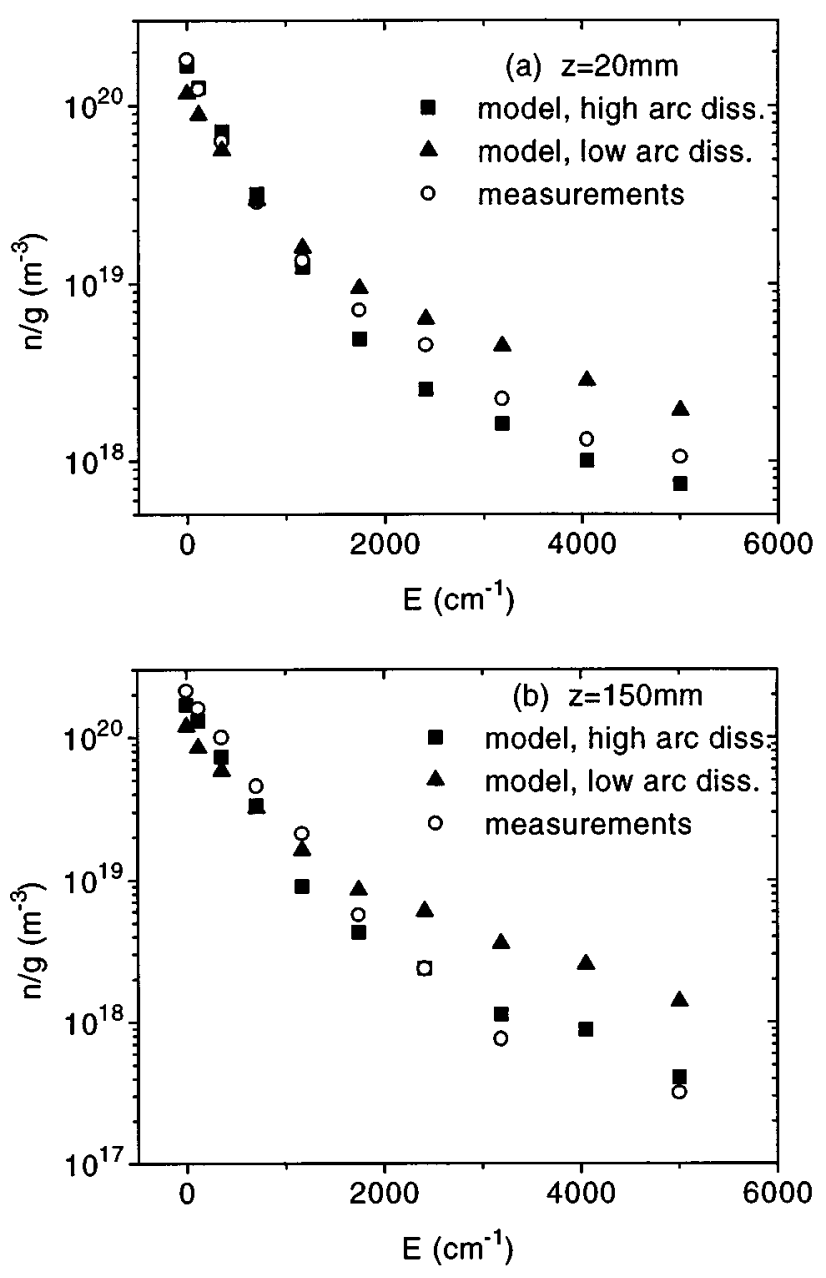

FIG. 10. The results of the quasi-one-dimensional model compared to the rotational distributions in $v=0$, at two axial positions, $z=20 \mathrm{~mm}$ (a) and $z=150 \mathrm{~mm}$ (b). The squares denote the model with a $70 \%$ dissociation degree in the arc, the triangles denote the model with a low dissociation degree $(10 \%)$ in the arc. The latter has been added to emphasize the fact that a substantial dissociation degree inside the cascaded arc has to be assumed in order to explain the measurements. The plasma is a freely expanding pure hydrogen jet (cf. Fig. 8).

periphery of the plasma, even though they appear to be rather cool. The partial $\mathrm{H}_{2}$ pressure in the periphery of the jet equals the seed fraction of $\mathrm{H}_{2}$, whether it be injected in the arc or in the vessel. A recirculation pattern in the vacuum vessel is thought to be responsible for the transport of (wallassociated) molecules into the plasma.

The measurements on pure hydrogen confirm the older depolarization Rayleigh scattering [2] and active probe [26] measurements, as all show a marginal dissociation degree in the vessel (i.e., the hydrogen pressure is equal to the background pressure within the experimental errors). An exact comparison between the two methods is difficult, as depolarization Rayleigh scattering is a crossed-beam experiment with a very high spatial resolution, whereas CARS has a poorer resolution in the longitudinal direction. The low dissociation degree in the vessel, in combination with the high dissociation degree in the arc, must be caused by a wallassociation process being dominant. 
The axial dependencies of $T_{\text {rot }}$ and $T_{\text {vib }}$ show no stationary shock front, which is quite evident in pure argon and diluted $\mathrm{Ar}-\mathrm{H}_{2}$ mixtures [4,5].

For the expanding pure hydrogen plasma, only states up to $v=2$ can be detected. De Graaf et al. [3] assume a total $v=4$ density of about $10^{19} \mathrm{~m}^{-3}$ in order to explain the anomalous recombination in a very similar pure hydrogen expanding plasma. The highly excited molecules were thought to originate from the vessel walls [27]. The hot molecules are necessary to make the reaction

$$
\mathrm{H}_{2}^{v, J}+\mathrm{H}^{+} \rightarrow \mathrm{H}_{2}{ }^{+}+\mathrm{H}
$$

exothermic (the energy deficit is around $2 \mathrm{eV}$ ). The formed $\mathrm{H}_{2}{ }^{+}$can either react with $\mathrm{H}_{2}$ in order to form $\mathrm{H}_{3}{ }^{+}$or with an electron in a very fast dissociative recombination reaction. The formed $\mathrm{H}_{3}{ }^{+}$can also dissociatively recombine, so either process will lead to a very effective ionization loss, once the $\mathrm{H}_{2}{ }^{+}$is formed.

The CARS measurements in this paper, however, clearly show the absence of large amounts of highly excited molecules around the plasma (where a $T_{\text {vib }}$ of about $1100 \mathrm{~K}$ is observed). At the measured $T_{\text {vib }}$ 's in the plasma, a $v=4$ density for the free expanding pure hydrogen jet can be estimated to be around $5 \times 10^{16} \mathrm{~m}^{-3}$ for axial positions between $z=40$ and $150 \mathrm{~mm}$. This discrepancy of more than two orders of magnitude is not well understood, but a different mechanism may have to be found in order to explain the observed ionization loss. Examples could be the expansion speed of the $\mathrm{H}^{+}$leaving the arc (translational energy), a pronounced importance of highly excited rotational states (e.g., a plateau in the rotational population below the detectivity of the CARS setup), or a combination of the mentioned possibilities of energy input (translational, rotational, and vibrational energy).

A similar problem arises for the supposedly $\mathrm{H}^{-}$rich magnetized hydrogen plasma: in this case, even higher excitation is necessary to make reaction (7) effective. But again, only low $T_{\text {vib's }}$ 'se observed (Fig. 9), which lead to estimated $v=4$ densities of a few times $10^{16} \mathrm{~m}^{-3}$. A strong plateau in the rotational population (just below the detection limit of the CARS setup) could be of influence here. If the presence of high densities of negative ions can be positively verified, however, it is clear that the standard "dissociative attachment involving vibrationally excited molecules" explanation cannot account for the production of negative ions.

The simple rate model of a two-population $\mathrm{H}_{2}$ gas seems to be able to describe the observed rotational distributions rather well for the freely expanding hydrogen jet. In this view, hot arc $\mathrm{H}_{2}$ at $3000 \mathrm{~K}$ (representing the $30 \%$ fraction that is not dissociated) expands into the vessel and relaxes to cold $(600 \mathrm{~K})$ hydrogen gas. The latter is supposed to constitute an infinite reservoir of cool gas. The model shows that the assumption of a high dissociation degree in the arc is necessary in order to explain the rotational state distribution.

\section{ACKNOWLEDGMENTS}

This work is supported by the Netherlands Technology Foundation (STW). The skillful technical assistance of H. M. M. de Jong, A. B. M. Hüsken, and M. J. F. van de Sande is gratefully acknowledged. Marco Box, Paulo Freire, Marc Beurskens, and Leon Jacobs are thanked for their help during the measurements. The people at ONERA Palaiseau (JeanPierre Taran, Michel Péalat, Michel Lefebvre, and Bruno Scherrer) have made a significant contribution through the many helpful discussions about the practical and theoretical aspects of CARS. The useful discussions with J. Uhlenbush, P. Jauernik, and S. Hädrig of Düsseldorf University, as well as with Richard van de Sanden (Eindhoven University) and Nader Sadeghi (Grenoble University) are gratefully acknowledged.
[1] See, for example, A. J. M. Buuron, G. J. Meeusen, J. J. Beulens, M. C. M. van de Sanden, and D. C. Schram, J. Nucl. Mater. 200, 430 (1993); R. J. Severens, G. J. H. Brussaard, M. C. M. van de Sanden, and D. C. Schram, Appl. Phys. Lett. 67, 491 (1995).

[2] R. F. G. Meulenbroeks, D. C. Schram, L. J. M. Jaegers, and M. C. M. van de Sanden, Phys. Rev. Lett. 69, 1379 (1992).

[3] M. J. de Graaf, R. Severens, R. P. Dahiya, M. C. M. van de Sanden, and D. C. Schram, Phys. Rev. E 48, 2098 (1993).

[4] M. C. M. van de Sanden, J. M. de Regt, G. M. Janssen, J. A. M. van der Mullen, B. van der Sijde, and D. C. Schram, Rev. Sci. Instrum. 63, 3369 (1992).

[5] R. F. G. Meulenbroeks, A. J. van Beek, A. J. G. van Helvoort, M. C. M. van de Sanden, and D. C. Schram, Phys. Rev. E 49, 4397 (1994).

[6] R. F. G. Meulenbroeks, R. A. H. Engeln, M. C. M. van de Sanden, J. A. M. van der Mullen, and D. C. Schram, in Proceedings of the International Symposium on Plasma Chemistry, edited by J. V. Meberlein, D. W. Ernie, and J. T. Roberts (University of Minnesota, Minneapolis, 1995).
[7] R. F. G. Meulenbroeks, R. A. H. Engeln, M. N. A. Beurskens, R. M. J. Paffen, M. C. M. van de Sanden, J. A. M. van der Mullen, and D. C. Schram, Plasma Sources Sci. Technol. 4, 74 (1995).

[8] A. J. Druet and J.-P. E. Taran, Prog. Quant. Electron. 7, 1 (1981).

[9] J.-P. E. Taran, CARS Spectroscopy in Applied Laser Spectroscopy, edited by W. Demtröder and M. Inguscio (Plenum, New York, 1990).

[10] M. Péalat, J.-P. E. Taran, and J. Taillet, J. Appl. Phys. 52, 2687 (1981).

[11] M. Péalat, M. Lefebvre, and J.-P. E. Taran, Phys. Rev. A 38, 1948 (1988).

[12] M. Péalat, M. Lefebvre, B. Scherrer, and J.-P. E. Taran (private communication).

[13] D. A. Long, Raman Spectroscopy (McGraw-Hill, London, 1977).

[14] J. Rychlewski, Mol. Phys. 41, 833 (1980).

[15] K. Chen, M. Chuang, C. M. Penney, and W. F. Banholzer, J. Appl. Phys. 71, 1485 (1992). 
[16] R. F. G. Meulenbroeks, D. C. Schram, M. C. M. van der Sanden, and J. A. M. van der Mullen, Phys. Rev. Lett. (to be published).

[17] M. C. M. van de Sanden, Z. Qing, D. K. Otorbaev, M. J. de Graaf, J. C. A. Wevers, and D. C. Schram, in Proceedings of the 5th European Workshop on the Production and Application of Light Negative Ions, Dublin, 1993, edited by M. Hopkins and S. Fahy (Dublin City University, Dublin, 1993), Vol. 41.

[18] See, for example, D. A. Greenhalgh, Quantitative CARS Spectroscopy, in Advances in Non-Linear Spectroscopy, edited by R. J. H. Clark and R. E. Hester (Wiley, New York, 1988); Combusting Flow Diagnostics, edited by D. F. G. Durão, M. V. Heitor, J. H. Whitelaw, and P. O. Witze, NATO Advanced Study Institute Series (Kluwer Academic, Amsterdam, 1992).

[19] M. Bacal, A. M. Bruneteau, W. H. Graham, G. W. Hamilton, and M. Nachman, J. Appl. Phys. 52, 1247 (1981).

[20] M. J. de Graaf, Z. Qing, H. W. A. Tolido, M. C. M. van de Sanden, and D. C. Schram, J. High Temp. Chem. Process. Suppl. to No. 3, 11 (1992).

[21] Alan Garscadden and Rajesh Nagpal, Plasma Sources Sci. Technol. 4, 268 (1995).

[22] A. E. DePristo, S. D. Augustin, R. Ramaswamy, and H. Rabitz, J. Chem. Phys. 71, 580 (1979); J. Derouard and N. Sadeghi, Chem. Phys. 88, 171 (1988).

[23] D. L. Thompson, J. Chem. Phys. 75, 1829 (1981).

[24] S. Green, J. Chem. Phys. 62, 2271 (1975).

[25] MAPLE is a registered trademark of 766884 Ontario inc. Waterloo Maple Software.

[26] D. K. Otorbaev, M. J. de Graaf, M. C. M. van de Sanden, and D. C. Schram, Contrib. Plasma Phys. 35, 195 (1995).

[27] B. Jackson and M. Persson, J. Chem. Phys. 96, 2378 (1992). 